\title{
EXISTENCE OF POSITIVE SOLUTIONS FOR QUASILINEAR ELLIPTIC SYSTEMS WITH SOBOLEV CRITICAL EXPONENTS
}

\section{XiAOYAN CAO AND ZUODONG YANG}

Abstract. In this paper, we consider the existence of positive solutions to the following problem

$$
\left\{\begin{array}{l}
-\operatorname{div}\left(|\nabla u|^{p-2} \nabla u\right)=\frac{\partial F}{\partial u}(u, v)+\varepsilon^{p-1} g(x) \text { in } \Omega, \\
-\operatorname{div}\left(|\nabla v|^{q-2} \nabla v\right)=\frac{\partial F}{\partial v}(u, v)+\varepsilon^{q-1} h(x) \text { in } \Omega, \\
u, v>0 \text { in } \Omega, \\
u=v=0 \text { on } \partial \Omega,
\end{array}\right.
$$

where $\Omega$ is a bounded smooth domain in $\mathbb{R}^{N} ; F \in C^{1}\left(\left(\mathbb{R}^{+}\right)^{2}, \mathbb{R}^{+}\right)$is positively homogeneous of degree $\mu ; g, h \in C^{1}(\bar{\Omega}) \backslash\{0\} ;$ and $\varepsilon$ is a positive parameter. Using sub-supersolution method and comparison principle, we prove the existence of positive solutions for the above problem.

Mathematics subject classification (2010): 35J25, 35J60.

Keywords and phrases: elliptic systems, subsolutions, supersolutions, comparison principle.

\section{REFERENCES}

[1] C. O. Alves, D. C. De Morais Filho And M. A. S. Souto, On system of elliptic equatins involving subcritical or critical Sobolev exponents, Nonlinear Anal., 42 (2000), 777-787.

[2] H. Amann, Fixed point equations and nonlinear eigenvalue problems in ordered Banach spaces, SIAM Rev., 18 (1976), 620-709.

[3] A. Ambrosetti and P. H. Rabinowitz, Dual variational methods in critical point theory and applications, J. Funct. Anal., 14 (1973), 347-381.

[4] G. Astrita And G. Marrucci, Principles of non-Newtonian fluid mechanics, McGraw-Hill, 1974.

[5] T. BARTSCH AND Y. X. GuO, Existence and nonexistence results for critical growth polyharmonic elliptic systems, J. Differential Equations, 200, 2 (2006), 531-543.

[6] H. BREZIS AND E. LIEB, A relation between pointwise convergence of functions and convergence of functionals, Proc. Am. Math. Soc., 88 (1983), 486-499.

[7] H. Brezis AND L. NiRENBerg, Positive solutions of nonlinear elliptic equations involving critical Sobolev exponents, Comm. Pure Appl. Math., 36, 4 (1983), 437-477.

[8] C. M. CHU AND C. L. TANG, Existance and multiplicity of positive solutions for semilinear elliptic systems with Sobolev critical exponents, Nonlinear Anal., 71 (2009), 5118-5130.

[9] Ph. Clément, D. G. De Figueiredo And E. Mitidieri, Positive solutions of semilinear elliptic systems, Comm. Partial Differential Equations, 17, 5-6 (1992), 923-940.

[10] Ph. Clément, R. Manasevich and E. Mitidieri, Positive solutions for a quasilinear system via blow up, Comm. Partial Differential Equations, 12, 18 (1993), 2071-2106.

[11] F. Cirstea AND V. D. RAdulescu, Entire solutions blowing up at infinity for semilinear elliptic systems, J. Math. Pures Appl., 81 (2002), 827-846.

[12] Q. Y. DAI AND Y. G. GU, Positive solutions for non-homogeneous semilinear elliptic equations with data that changes sign, Proc. Roy. Soc. Edinburgh Sect. A, 133, 2 (2003), 297-306.

[13] Q. Y. Dai And L. H. PEng, Necessary and sufficient conditions for the existence of nonnegative solutions of inhomogeneous p-Laplace equation, Acta Math. Sci. Ser. B Engl. Ed., 27, 1 (2007), 34 56.

[14] Q. Y. DAI AND J. F. YAng, Positive solutions of inhomogeneous elliptic equations with indefinite data, Nonlinear Anal., 58, 5-6 (2004), 571-589. 
[15] R. DALmasso, Existence and uniqueness of positive solutions of semilinear elliptic system, Nonlinear Anal., 39 (2000), 559-568.

[16] J. I. Diaz, J. M. Morel AND L. Oswald, An elliptic equation with singular nonlinearity, Comm. Partial Differential Equations, 12, 12 (1987), 1333-1344.

[17] E. DiBenedetto, $C^{1+\alpha}$ local regularity of weak solutions of degenerate elliptic equations, Nonlinear Anal., 7, 8 (1983), 827-850.

[18] A. Edelson, Entire solutions of singular elliptic equations, J. Math. Anal. Appl., 139 (1989), $523-$ 532.

[19] J. R. Esteban AND J. L. VAzQuez, On the equation of turbulent filteration in one-dimensional porous media, Nonlinear Anal., 10 (1982), 1303-1325.

[20] P. L. Felmer, R. MANASEVICh AND F. DE Thelin, Existence and uniqueness of positive solutions for certain quasilinear elliptic system, Comm. Partial Differential Equations, 17 (1992), 2013-2029.

[21] J. Fleckinger, J. HeRnNDEZ AND F. D. Thlin, On maximum principles and existence of positive solutions for some cooperative elliptic systems, Differential Integral equations, 8 (1995), 69-85.

[22] J. Graham-EAGLE, Monotone methods for semilinear elliptic equations in unbounded domains, J. Math. Anal. Appl., 137, 1 (1989) 122-131.

[23] Z. Guo, Existence of positive radial solutions for a class of quasilinear elliptic systems in annular domains, Chinese J. Contemp. Math., 17, 4 (1996), 337-350

[24] Z. Guo, On the number of positive solutions for quasilinear elliptic eigenvalue problems, Nonlinear Anal., 27, 2 (1996), 229-247.

[25] Z. GuO, Some existence and multiplicity results for a class of quasilinear elliptic eigenvalue problems, Nonlinear Anal., 18 (1992), 957-971.

[26] Z. GUO AND J. R. L. WeBB, Uniqueness of positive solutions for quasilinear elliptic equations when a parameter is large, Proc. Roy. Soc. Edinburgh Sect. A, 124, 1 (1994), 189-198.

[27] D. D. HAI, Positive solutions of quasilinear boundary value problems, J. Math. Anal. Appl., 217 (1998), 672-686.

[28] P. G. HAN, Multiple positive solutions of nonhomogeneous elliptic systems involving critical Sobolev exponents, Nonlinear Anal., 64, 4 (2006), 869-886.

[29] A. S. Kalashnikov, A nonlinear equation arising in the theory of nonlinear filtration, Trudy Sem. Petrovsk., 4 (1978), 137-146.

[30] T. KUSANO AND C. A. SWANSON, Entire positive solutions of singular semilinear elliptic equations, Japan. J. Math. (N.S.), 11 (1985), 145-155.

[31] A. V. LAIR AND A. W. WOOD, Existence of entire large positive solutions of semilinear elliptic systems, J. Differential Equations, 164, 2 (2000), 380-394.

[32] G .M. Lieberman, Boundary regularity for solutions of degenerate elliptic equations, Nonlinear Anal., 12, 11 (1988), 1203-1219.

[33] C. L. LIU AND Z. YANG, Existence of large solutions for quasilinear elliptic problems with a gradient term, Appl. Math. Comput., 192 (2007), 533-545.

[34] L. K. MARTINSON AND K. B. PAVLOV, Unsteady shear flows of a conducting fluid with a rheological power law, Magnitnaya Gidrodinamika, 2 (1971), 50-58.

[35] Q. Miao And Z. YAng, Bounded Positive Entire Solutions of Singular p-Laplacian Equations, Nonlinear Anal., 69 (2008), 4380-4391.

[36] E. Mitidieri, A Rellich type identity and applications, Comm. Partial Differential Equations, 18, 1-2 (1993), 125-151.

[37] E. MitidiERI, Nonexistence of positive solutions of semilinear elliptic system in $\mathbb{R}^{N}$, Differential Integral Equations, 9 (1996), 465-479.

[38] E. Mitidieri, G. SWEers AND R. VAn DER Vorst, Nonexistence theorems for systems of quasilinear partial differential equations, Differential Integral Equations, 8 (1995), 1331-1354.

[39] W. M. NI AND J. SERRIN, Nonexistence theorems for singular solutions of quasilinear partial differential equations, Comm. Pure Appl. Math., 39, 2 (1986), 379-399.

[40] A. OGATA, On bounded positive solutions of nonlinear elliptic boundary value problems in an exerior domain, Funkcial. Ekvac., 17 (1974), 207-222.

[41] C. V. PAO, Positive solutions of a nonlinear boundary value problem of parabolic type, J. Differential Equations, 22 (1976), 145-163. 
[42] L. A. PELETIER AND R. VAN DER VORSt, Existence and non-existence of positive solutions of nonlinear elliptic systems and the biharmonic equations, Differential Integral Equations, 54 (1992), 747767.

[43] J. SERRIN AND H. ZOU, Cauchy-Liouville and universal boundedness theorems for quasilinear elliptic equations and inequalities, Acta Math., 189 (2002), 79-142.

[44] C. A. Swanson, The best Sobolev constant, Appl. Anal., 47, 4 (1992), 227-239.

[45] G.Tarantello, On nonhomogeneous elliptic equations involving critical Sobolev exponent, Ann. Inst. H. Poincaré Anal. Non Linéaire, 9, 3 (1992), 281-304.

[46] J. VAZQUEZ, A strong maximum principle for some quasilinear elliptic equations, Appl. Math. Optim., 12, 3 (1984), 191-202.

[47] X. B. Jin AND C. Z. CHI, Solvability of singular quasilinear elliptic equation, Chinese Ann. Math. Ser. A, 20, 1 (1999), 117-128.

[48] Z. YANG, Existence of explosive positive solutions of quasilinear elliptic equations, Appl. Math. Comput., 177, 2 (2006), 581-588.

[49] Z. YANG, Existence of positive entire solutions for singular and non-singular quasi-linear elliptic equation, J. Comput. Appl. Math., 197, 2 (2006), 355-364.

[50] Z. YANG, On the existence of multiple positive entire solutions for a class of quasilinear elliptic equations, Int. J. Math. Math. Sci., 2006, 19 pp.

[51] Z. YANG, Existence of entire explosive positive radial solutions for a class of quasilinear elliptic systems, J. Math. Anal Appl., 288, 2 (2003), 768-783.

[52] Z. YANG, Existence of positive bounded entire solutions for quasilinear elliptic equations, Appl. Math. Comput., 156, 3 (2004), 743-754.

[53] Z. YANG AND Q. LU, Existence and multiplicity of positive entire solutions for a class of quasilinear elliptic equation, J. Beijing Univ. Aeronaut. Astronaut., 27 (2001), 217-220.

[54] Z. YANG AND Q. LU, Non-existence of positive radial solutions for a class of quasilinear elliptic system, Commun. Nonlinear Sci. Numer. Simul., 5 , 4 (2000), 184-187.

[55] Z. YANG AND H. YANG, A priori for a quasilinear elliptic P.D.E. non-positone problems, Nonlinear Anal., 43, 2 (2001), 173-181.

[56] Z. YANG AND Z. GUO, Existence of positive radial solutions and entire solutions for quasilinear singular boundary value problems, Ann. Differential Equations, 12, 2 (1996), 243-251.

[57] K. YosidA, Functional Analysis, Springer-Verlag, Berlin, 1980. 